\title{
eReferrals: Why are we still faxing?
}

Christopher A Hughes, Penny Allen, Michael Bentley

\section{Background and objective eReferrals have the potential to be a transformative technology in the healthcare space. This study explored attitudes, behaviours and barriers to eReferral use and electronic communication in general.}

\section{Method}

A survey of doctors and allied health staff was undertaken in north-west Tasmania. Data were analysed using descriptive statistics and thematic analysis.

\section{Results}

The response rate was $57 \%(n=204)$. For $80 \%(n=164)$ of respondents, fax or post was the main method of sending letters to other healthcare professionals, and $72 \%(n=147)$ wanted to increase the number of letters sent and received electronically. Barriers and enablers to eReferral use included peer behaviour, software factors, security issues and workplace culture.

\section{Discussion}

Somewhat ironically, the key barrier to eReferral use was peers not using eReferrals. A greater emphasis on software usability and interoperability is required. Despite eReferrals being promoted as the more secure alternative, security remains a key concern. Workplaces could influence adoption by encouraging eReferral use.
AUSTRALIAN general practitioners (GPs) send a lot of correspondence, and this is increasing. ${ }^{1}$ In 2014-15 they made 15.9 referrals per 100 patient encounters, ${ }^{2}$ and sent 9.7 million more referrals than in 2005-06. ${ }^{1}$

eReferrals are electronic letters sent between healthcare professionals via secure means. ${ }^{3}$ In Australia, several secure messaging services offer this functionality. In 2016, the four major providers had 117,000 registered user accounts, ${ }^{4}$ and $40 \%$ of Australian GPs sent and received information via secure message delivery. ${ }^{5}$ eReferrals cannot currently be sent from one secure message service to another. ${ }^{6}$

The Practice Incentives Program (PIP) eHealth Incentive is administered by the federal Department of Health, and pays up to $\$ 50,000$ per year to general practices that use telehealth, including eReferrals. ${ }^{7}$ In $2013,71 \%$ of general practices in north-west Tasmania claimed this incentive payment. ${ }^{8}$ Commonly postulated benefits of eReferrals include:

- reduced waiting times ${ }^{9}$

- referral tracking (data collection) $)^{10}$

- improved referral quality ${ }^{11}$

- opportunity for eConsultation (pre-consultation dialogue between author and recipient $)^{12}$

- cost reduction. ${ }^{13}$

The most commonly reported limitation of eReferrals is the potential to negatively affect the user's workflow. ${ }^{14}$

This research was prompted by the primary investigator's perception that there is little eReferral use among GPs in north-west Tasmania, a region that had 129 GPs in $2014 \cdot{ }^{15}$ Research into eReferrals has largely been analyses of various eReferral implementations. There is little research into the users' perspective, particularly:

- What do they want from an eReferral service?

- What prevents them from using eReferrals?
- What are their current behaviours in the eReferral space?

Similarly, there are no data on eReferral usage in regional Australia. The objective of this study was to explore attitudes, behaviours and barriers to the uptake of eReferrals and electronic communication generally among healthcare professionals in north-west Tasmania.

\section{Method}

Approval for this study was obtained from the Tasmania Social Sciences Human Research Ethics Committee (reference number: Hoo15342).

\section{Design}

A literature review was undertaken by the primary investigator (PubMed search; English language; full text available; titles only; search terms: ereferrals, ereferral, e-referrals, e-referral, electronic referrals, electronic referral, electronic booking, secure messaging, secure messages, secure message). The reference lists of highly relevant articles were also searched for relevant articles. No survey that had undergone validity and reliability testing was identified on the topic of eReferral behaviours and attitudes. Those surveys identified were designed to capture data before and after specific eReferral implementations.

A draft survey was developed to capture information on attitudes and behaviours to electronic modes of communication in general, and eReferrals specifically. The draft survey was piloted and underwent face validation with six healthcare professionals in southern Tasmania. Pilot participants completed the survey within an average of four minutes, and reported that it was an appropriate length and easy to complete. The survey was adopted as the final survey, with minor changes to the wording of two questions following feedback from pilot participants. 


\section{Participants}

A list of 356 healthcare professionals working in north-west Tasmania was compiled using data from the Tasmanian Health Directory, ${ }^{16}$ the National Health Services Directory, ${ }^{17}$ and the Saunders Street Clinic practice management software address book. Healthcare professions included were GPs, general practice registrars, allied health professionals (eg dietitians, occupational therapists, physiotherapists, podiatrists, psychologists), and non-GP specialists (eg general surgeons, obstetricians, psychiatrists, paediatricians, general physicians). North-west Tasmania was defined by the Census of Tasmanian general practices. ${ }^{2}$ If insufficient data were available, the healthcare professional was telephoned to request further contact information.

In January 2016, an invitation email was sent to invitees $(n=338)$, followed by a fax $(n=342)$. A reminder email was sent to non-responders in February $2016(\mathrm{n}=135)$. The survey was closed on 1 March 2016.

\section{Data collection}

Survey response data were gathered anonymously. Healthcare professionals were invited to participate via an online survey or return the survey via email, fax or post. They were also given the option to request a phone interview or in-person interview. The survey collected information on eReferrals and electronic modes of communication in general.

\section{Data analysis}

Data from the online survey were downloaded into Microsoft Excel. Quantitative data were imported into Stata14 for descriptive analysis. Categorical data were investigated to determine the number and percentage of participants in each subcategory. Crosstabs with chi-square tests (or Stuart-Maxwell tests where appropriate) were used to investigate the association between professional role and current eReferral use, and intended future changes in eReferral use. Standardised residuals of crosstabs were investigated to identify cells in the crosstab where counts exceeded expected counts.
One-way analysis of variance (ANOVA) was used to investigate the association between age and current eReferral use, and age and desire to increase eReferral use. All tests were two-sided and differences were accepted as significant at $P<0.05$.

The survey included two open-ended questions on barriers and enablers of eReferrals:

1. What is the greatest barrier to your use of eReferrals?

2. What would make you more likely to use eReferrals?

The primary investigator analysed the free text data from the two openended questions to identify common themes and subthemes. The other investigators separately reviewed the free text categorisation for accuracy and to determine agreement with the identified themes.

\section{Results}

\section{Demographics}

A total of 204 healthcare professionals responded to the invitation to complete the survey (57\% response rate).

Professions that responded were allied health professionals $(n=64 ; 33 \%)$, GPs ( $\mathrm{n}=51 ; 27 \%)$, non-GP specialists $(\mathrm{n}=30 ; 16 \%)$, general practice registrars $(\mathrm{n}=15,7 \%)$, nurses $(\mathrm{n}=16 ; 8 \%)$, practice managers $(\mathrm{n}=14 ; 7 \%)$, and 'Other' $(\mathrm{n}=2 ; 1 \%)$. Profession data were missing for 12 respondents (6\%). The response rate among general practice registrars was $100 \%$, nurses $80 \%$, allied health professionals $49 \%$, non-GP specialists $49 \%$, GPs $36 \%$ and practice managers $34 \%$.

The majority of respondents $(n=142$; $69.5 \%$ ) completed the survey online; the remaining 62 respondents sent their surveys by fax $(n=49 ; 24.0 \%)$, email $(n=5 ; 2.5 \%)$ or post $(n=3 ; 1.5 \%)$, or completed them via in-person interview $(\mathrm{n}=5 ; 2.5 \%)$. The mean age of respondents was 48 years (standard deviation [SD]: 11.6; range: $\left.24^{-76}\right)$, and $53 \%(\mathrm{n}=107)$ were female and $42 \%(n=86)$ male. Gender data were missing for 11 respondents (5\%). 'Practice management software' was used by $64 \%$ $(n=131)$ of respondents, $28 \%(n=58)$ indicated they did not use 'practice management software', $5 \%(\mathrm{n}=10)$ did not answer this question and $3 \%(\mathrm{n}=5)$ indicated they were 'Unsure'.

\section{Primary mode of communication}

Fax was elected as the primary mode of sending letters by $53 \%$ of respondents $(\mathrm{n}=109), 27 \%$ elected post $(\mathrm{n}=55), 12 \%$ elected eReferrals $(n=25), 6 \%$ elected email $(n=12), 1 \%$ elected 'Other' $(n=2)$ and $1 \%$ were 'Unsure' $(\mathrm{n}=1)$. Among GPs and general practice registrars, 92\% $(n=61)$ elected fax as the primary mode of sending letters, $5 \%(n=3)$ elected post, $3 \%(n=2)$ elected eReferrals and none elected email (Table 1 ).

Traditional methods of communication (ie post, fax) were chosen by $80 \%(n=164)$ of respondents as the primary mode of sending letters, and $82 \%(n=167)$ as the primary mode of receiving letters. By contrast, electronic communications (ie eReferrals, email) were elected by $18 \%$ $(\mathrm{n}=37)$ as the primary mode of sending letters, and $10 \%(n=20)$ as the primary mode of receiving letters (Table 1 ).

\section{Electronic communication}

Respondents were asked to estimate the proportion of letters they send and receive by electronic means. Thirty-one per cent $(n=64)$ of respondents sent no letters electronically, $43 \%(\mathrm{n}=88)$ sent some, $2 \%(n=5)$ sent half, $15 \%(n=31)$ sent a majority and $1 \%(n=3)$ sent all letters electronically. The remaining respondents were 'Unsure' $(3 \%, \mathrm{n}=7)$, did not answer $(1 \% ; \mathrm{n}=3)$ or indicated an 'Other' proportion $(1 \% ; n=3)$.

Among GPs and general practice registrars, $55 \%(\mathrm{n}=36)$ sent no letters electronically, $32 \%(n=21)$ sent some, $2 \%$ $(n=1)$ sent half, $3 \%(n=2)$ sent most, and no one sent all letters electronically. The remaining respondents were 'Unsure' $(6 \% ; n=4)$ or indicated an 'Other' proportion $(3 \% ; n=2)$.

Practice managers were more likely to send and receive a higher proportion of letters electronically than other professions $\left(\chi^{2}(3)=53.1 ; P<0.001\right)$. The age of the respondent was not associated with the proportion of letters sent and received electronically $(F(2,175)=0.008 ; P=0.99)$. 
Table 1. Primary mode of communication

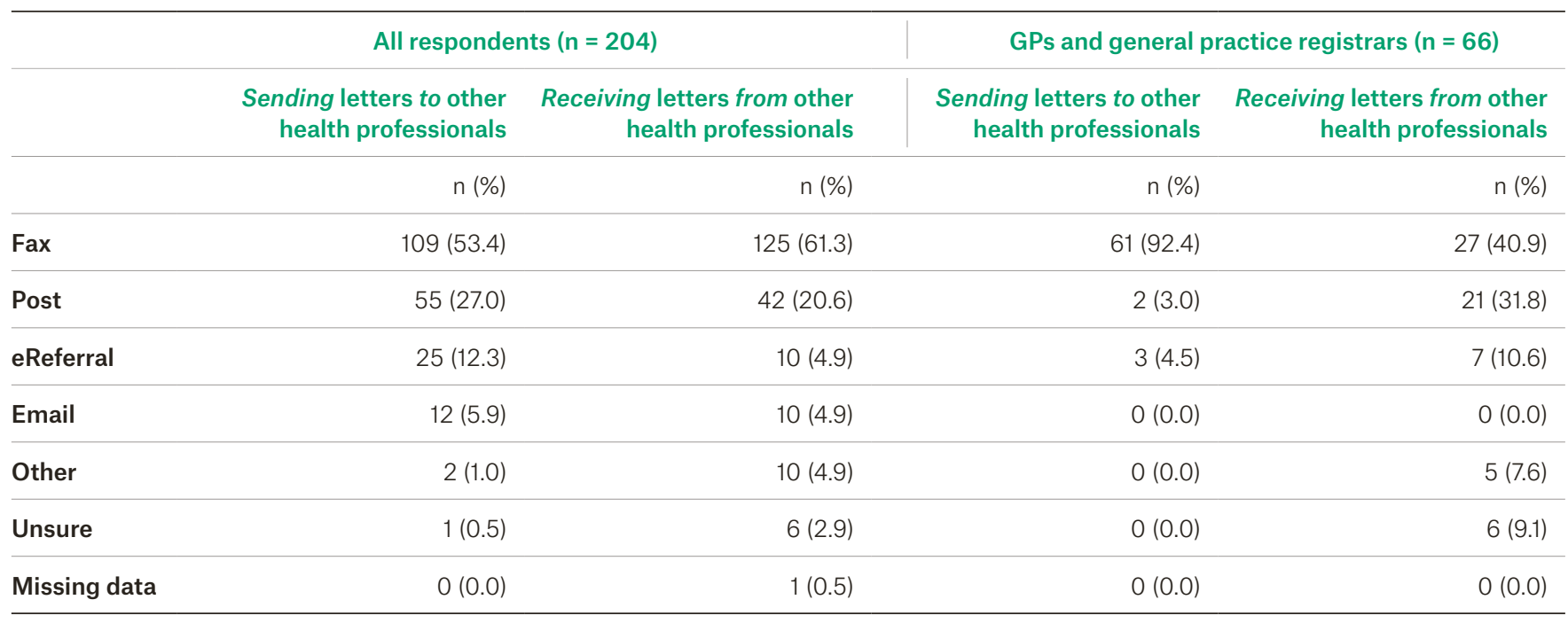

\section{Desire to increase electronic communications}

A desire to increase the number of letters sent and received electronically was indicated by $72 \%$ of respondents $(n=147)$. By contrast, $10 \%$ of respondents indicated they did not want to send and receive more letters electronically $(\mathrm{n}=20), 16 \%$ were undecided $(\mathrm{n}=33)$ and $2 \%$ did not enter a response to this question $(n=4)$. Among GPs and general practice registrars, $80 \%(n=53)$ wanted to increase the number of letters they sent and received electronically, $6 \%(n=4)$ did not desire an increase and $14 \%$ $(n=9)$ were undecided. Non-GP specialists were less likely to want to increase the number of letters they sent and received electronically $\left(\chi^{2}(4)=137.7\right.$; $P<0.001)$. Age $(F(2,178)=2.2 ; P=0.11)$ and access to practice management software were not associated with the desire to increase the number of letters sent and received electronically $\left(\chi^{2}(2)=2.1, P=0.34\right)$.

\section{Barriers and enablers to using eReferrals}

The open-ended question regarding the greatest barrier to the use of eReferrals was answered by 187 participants (92\%). The open-ended question regarding what would make the respondent more likely to use eReferrals was answered by 179 participants $(88 \%)$. Thematic analysis of this data identified several barriers and enablers (Figure 1). The most common responses are discussed below.

\section{Peer behaviour}

The behaviour of peers was identified by $22 \%$ of respondents as a barrier to their use of eReferrals $(n=44)$. About one-quarter of respondents $(n=49 ; 24 \%)$ felt a change in peer behaviour would make them more likely to use eReferrals. Respondents reported frustration when an intended letter recipient was not available on their eReferral program. They also stated more generally that if other practitioners were using these services, they would be more inclined to do so as well. Some of the barriers around peer behaviour noted:

We don't have the barrier this end - GP practices are reluctant. They seem to be stuck in the old method of only faxing.

- Respondent 013

Availability and uptake-all specialists, GPs, the hospital etc would have to use the same system, which, once implemented, would be easier than faxing. However, I'm not sure how you would go about getting everyone on the same page! - Respondent 026

Some of the enablers around peer behaviour noted:
If there was a database of email addresses readily accessible by all health professionals... If more clinics and health professionals are made aware of it and using it. - Respondent 088

We want to use e referrals and cut out faxing. Needs more users on other end ... Many GP practices have referralnet, but a lot of GPs don't use it. - Respondent 106

\section{Software factors}

Software ease of use and interoperability was identified by $23 \%$ of respondents as a barrier to their use of eReferral $(n=46)$; $21 \%$ identified improved software and interoperability as a potential enabler $(n=43)$. Respondents were not happy with the way eReferral software interfaces with electronic medical records and the way it fits around their workflow. They also bemoaned the lack of interoperability between different eReferral systems. Some of the barriers around software factors noted:

Not user-friendly, too many steps to use ... ie, it takes about 6-10 clicks and 5 minutes just to send one letter! - Respondent 078

Each individual GP or service using their own system for referrals. Do not have capacity, time or resources to use multiple different systems. - Respondent 149 
Some of the enablers around software factors noted:

An easy-to-use software and dictation system that integrates with the current digital medical record system used by the hospital. - Respondent 047

\section{Programs that 'talk to each other' are} linked and streamline the process (ie straight from the patient's DMR without having to find GPs name and use other program). - Respondent 166

\section{Security}

Confidentiality and privacy fears were nominated by $22 \%$ of respondents as a barrier to eReferral use ( $n=45)$, and $14 \%$ identified improved security as a potential enabler of eReferral use $(n=29)$. Some of the barriers around security noted:
Confidentiality and security of information being sent. - Respondent 052

I believe from a recent documentation in-service that until absolute security can be assured, that the department of health still considers fax as the most appropriate form of communication/referral pathway at present for client sensitive information. - Respondent 201

Some of the enablers around security noted:

An acceptable encryption system or process that ensures patient information is secure.

- Respondent 005

We would use electronic media exclusively, if the privacy issue could be solved to the satisfaction of our medical defence insurance company. - Respondent $\mathrm{O} 21$

\section{Workplace culture}

The workplace not allowing eReferrals or not making them available was a reason stated by $20 \%$ of respondents $(n=40)$ as a barrier to their eReferral use. The workplace making eReferrals available was identified as an enabler by $12 \%$ of respondents $(n=25)$. Some of the barriers around workplace culture noted:

We have been advised by our department that we can only use electronic letter system to communicate with other health department employees. For outside correspondence, we have been requested to use a system [that] leaves a paper trail. - Respondent 099

Being advised by my superiors to only fax referrals. - Respondent 180

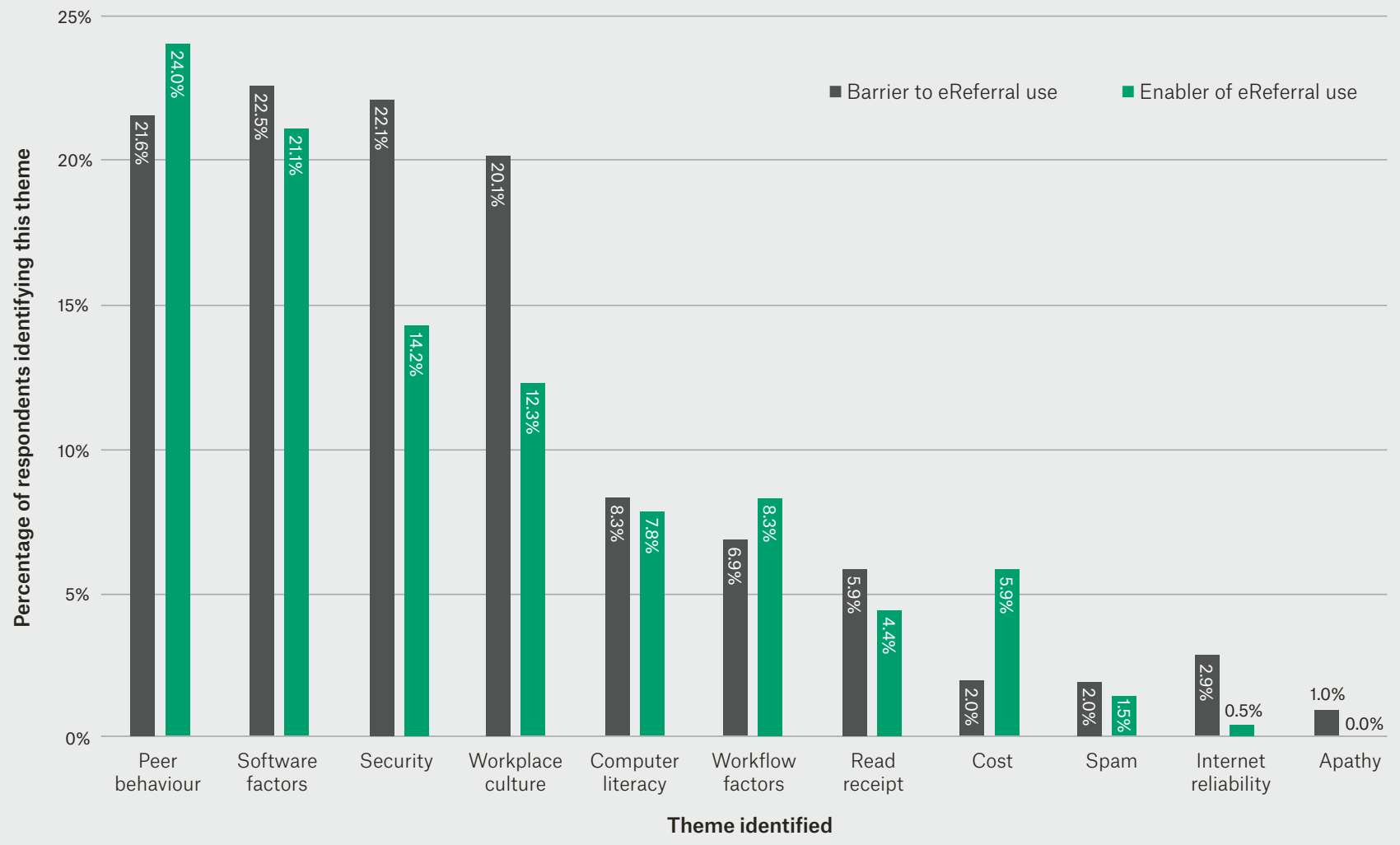

Figure 1. Barriers and enablers of eReferral use 
Some of the enablers around workplace culture noted:

If it was permitted in the hospital system, I would do it much more often. - Respondent 123

If it was made available to me again by my employer. I would much prefer to use an electronic system if available.

- Respondent 138

\section{Discussion}

This survey investigated attitudes, behaviours and barriers to eReferrals and electronic modes of communication in general. The study identified four important themes affecting eReferral uptake: peer behaviour, software factors, security and workplace culture.

The majority of respondents completed the survey via the online link, which demonstrates a readiness to use electronic modes of communication. In addition, most respondents indicated a desire to send and receive more letters electronically. By contrast, most respondents indicated that their current primary mode of communication was paper-based (ie fax, post). Nine out of 10 GPs and general practice registrars elected fax as their primary method of sending letters. Despite the high proportion of general practices in north-west Tasmania claiming the PIP eHealth Incentive, ${ }^{8}$ very few GPs and general practice registrars elected eReferrals as their main mode of sending correspondences. This incentive requires a practice to install a secure messaging service, use it where feasible, and have a written policy to encourage its use.

Ironically, the major reason for not using eReferrals was that one's peers were not using eReferrals or were not accessible on their current eReferral program. It can be expected that improved secure messaging service interoperability would alleviate this issue. Interoperability is a stated priority of both The Royal Australian College of General Practitioners (RACGP) and the Australian Digital Health Agency. ${ }^{5}$ In previous work, interoperability has been identified as a barrier to eReferral, ${ }^{18}$ but peer behaviour in a general sense has not.

In the time-poor environment of healthcare, it is important that the use of software is incorporated seamlessly into the healthcare professional's workflow. Two of the themes identified by our respondents, 'Software factors' and 'Workflow factors', can be considered together as 'Sociotechnical factors' (software design with an emphasis on usability). Other authors have commonly identified sociotechnical factors as a powerful influencer of eReferral uptake. ${ }^{19}$ Further research could investigate user satisfaction with eReferral software.

The security and privacy of eReferral systems were a concern for many respondents. This is an interesting point given that eReferral services are, in theory, the more secure alternative, and marketed by vendors this way. Even the name 'secure messaging service' seems designed to emphasise this concept. More education and reassurance is required regarding the security of eReferrals. Beliefs regarding security of eReferrals is a potential area for future research.

Our findings suggest that workplaces can influence eReferral uptake by encouraging eReferral use, or making it mandatory. If cost-effectiveness could be demonstrated, workplaces might be more inclined to encourage eReferral use. The cost-effectiveness of eReferrals is another potential area for future research.

Some respondents indicated a reluctance to embrace eReferrals because of perceived vulnerability to spam (ie unsolicited, non-relevant mail). Uncertainty over whether a letter has been received or read was another perceived barrier; however, integration of a 'Read receipt' could be a potential enabler. This last point is interesting, because knowing whether a letter has been received successfully is akey shortcoming of fax systems.

A strength of this project was its focus on the attitudes and behaviours of end users. This differs from most published research on eReferrals, which analysed specific eReferral implementations at various institutions. As a result, we have been able to find previously unidentified factors affecting eReferral uptake, including peer behaviour, security and workplace culture. Enablers of eReferral use identified by other authors include:

- iterative development - software deployment with planned modification based on formal feedback ${ }^{14}$

- a national standard approach/ government policy-setting ${ }^{13}$

- good information technology support and education. ${ }^{20}$

Barriers have included lack of funding for triaging and eConsultation, ${ }^{21}$ increased clinician workload ${ }^{22}$ and technical difficulties. ${ }^{23}$

Another key strength was the survey's inclusiveness of all members of the healthcare team. Authors and recipients must adopt the technology to allow it to function, so it is important to include both sides in the research. If we focused solely on GPs, we could expect a one-sided view. Practice managers are not authors or recipients of referrals, but may be considered powerful influencers of their colleagues' workflow and technology adoption, hence their inclusion in the survey.

\section{Study limitations}

This project was targeted to a small region of Tasmania and, therefore, may not be representative of the broader Australian community. The response rate of $57 \%$ is below the generally accepted minimal response rate of $70 \%$; however, response rates for medical practitioners are often less than $30 \% .{ }^{24}$ The response rate for general practice registrars was higher than for other professions, perhaps because the project was undertaken in conjunction with General Practice Training Tasmania as part of the primary author's Fellowship in Advanced Rural General Practice.

We are unable to comment on the reliability of the survey questions as we did not conduct reliability testing on the survey tool. The survey did undergo face validation, but we did not conduct validity testing of the draft survey. Although this is a limitation of the study, the survey design and wording were straightforward to reduce the risk of measurement bias. 


\section{Implications for general practice}

- Healthcare professionals would generally like to increase their electronic correspondence, yet most are currently faxing or posting their correspondence. Where eReferrals are used, the proportion is generally low.

- Behaviour of peers is the strongest influence on a healthcare professional's eReferral behaviour, and workplaces can play a key role.

- Interoperability of eReferral systems and software usability require development.

- Work is required to placate security and privacy concerns.

\section{Authors}

Christopher A Hughes FRACGP, FARGP, MBBS BMedSc, General Practitioner, Saunders Street Clinic, Wynyard, Tas; Clinical Lecturer, University of Tasmania; Medical Educator, General Practice Training Tasmania.fiftyfourhughes@gmail.com Penny Allen BA (Hons), MPH, PhD, Senior Research Fellow, Rural Clinical School, University of Tasmania Michael Bentley BSc, MA, DrPH, Research Officer, General Practice Training Tasmania; Adjunct Research Fellow, Southgate Institute for Health, Society and Equity, Flinders University; Senior Lecturer (Academic Status), College of Medicine and Public Health, Flinders University, SA

Competing interests: None.

Provenance and peer review: Not commissioned, externally peer reviewed.

Acknowledgments: The authors would like to acknowledge the assistance and advice of Dr Paul Fitzgerald, Colleen Cheek, Prof Bastian Seidel, Prof Michael Buist, Dr John Henshaw, and the Saunders Street Clinic.

\section{References}

1. Britt H, Miller GC, Henderson J, et al. A decade of Australian general practice activity 2005-06 to 2014-15. Sydney: Sydney University Press, 2015.

2. Britt H, Miller GC, Henderson J, et al. General practice activity in Australia 2014-15. Sydney: Sydney University Press, 2015.

3. Wootton R, Harno K, Reponen J. Organizational aspects of e-referrals. J Telemed Telecare 2003;9 Suppl 2:S76-79.

4. Scholefield A. Out with fax, in with secure messaging. Chatswood, NSW: Australian Doctor Cirrus Media, 11 October 2016. Available at www.australiandoctor.com.au/news/ latest-news/out-with-fax-in-with-securemessaging? $t=636250195433257724$ [Accessed 18 March 2017]

5. The Royal Australian College of General Practitioners. Views and attitudes towards technological innovation in general practice: Survey report 2016. East Melbourne, Vic: RACGP, 2017.

6. Australian Digital Health Agency. Fax machines on the way out for Australian healthcare. Sydney: Australian Digital Health Agency, 2017. Available at www.digitalhealth.gov.au/news-and-events/news/ fax-machines-on-the-way-out-for-australianhealthcare [Accessed 22 July 2017].

7. Department of Human Services. Practice Incentives Program. Canberra: DHS, 2017. Available at www.humanservices.gov.au/ health-professionals/services/medicare/practiceincentives-program [Accessed 8 July 2017].

8. Tye I. 2013 Census of Tasmanian general practices. Hobart: Tasmania Medicare Local Limited, 2014; p. 25.

9. Warren J, White S, Day KJ, Gu Y, Pollock M. Introduction of electronic referral from community associated with more timely review by secondary services. Appl Clin Inform 2011;2(4):546-64.

10. Batura D, Hashemzehi $T$, Lee $T$, et al. Beneath the tip of the iceberg: Using electronic referrals to map the unquantified burden of clinical activity in a urology service. Int Urol Nephrol 2016;48(11):1751-55.

11. Shaw LJ, de Berker DA. Strengths and weaknesses of electronic referral: Comparison of data content and clinical value of electronic and paper referrals in dermatology. Br J Gen Pract 2007;57(536):223-24.

12. Gu Y, Warren J, Orr M. The potentials and challenges of electronic referrals in transforming healthcare. N Z Med J 2014;127(1398):111-18.

13. Heimly V. Electronic referrals in healthcare: A review. Stud Health Technol Inform 2009;150:327-31.

14. Kim Y, Chen AH, Keith E, Yee HF Jr, Kushel MB Not perfect, but better: Primary care providers' experiences with electronic referrals in a safety net health system. J Gen Intern Med 2009;24(5):614-19.

15. Tye I. 2014 Census of Tasmanian general practices. Hobart: Tasmania Medicare Local Limited, 2015; p. 15.

16. Primary Health Tasmania. Tasmanian health directory. Hobart: Primary Health Tasmania, 2015. Available at www.primaryhealthtas.com.au/ tasmanian-health-directory [Accessed 17 Nov 2017].

17. Healthdirect Australia. National Health Services Directory. Sydney: Healthdirect Australia, 2016. Available at https://about.healthdirect.gov.au/ nhsd [Accessed 17 Nov 2017].

18. Liddy C, Hogel M, Blazkho V, Keely E. The current state of electronic consultation and electronic referral systems in Canada: An environmental scan. Stud Health Technol Inform 2015;209:75-83.

19. Esquivel A, Sittig DF, Murphy DR, Singh $H$. Improving the effectiveness of electronic health record-based referral processes. BMC Med Inform Decis Mak 2012;12:107.

20. Chambers EC, Wylie-Rosett J, Blank AE, et al. Increasing referrals to a YMCA-based diabetes prevention program: Effects of electronic referral system modification and provider education in federally qualified health centers. Prev Chronic Dis 2015;12:E189.

21. Tuot DS, Leeds K, Murphy EJ, et al. Facilitators and barriers to implementing electronic referral and/or consultation systems: A qualitative study of 16 health organizations. BMC Health Serv Res 2015;15:568.

22. Pagliari C, Donnan P, Morrison J, Ricketts I, Gregor P, Sullivan F. Adoption and perception of electronic clinical communications in Scotland. Inform Prim Care 2005;13(2):97-104.

23. Straus SG, Chen AH, Yee H Jr, Kushel MB, Bell DS Implementation of an electronic referral system for outpatient specialty care. AMIA Annu Symp Proc 2011;2011:1337-46.

24. Bonevski B, Magin P, Horton G, Foster M, Girgis A Response rates in GP surveys - Trialling two recruitment strategies. Aust Fam Physician 2011;40(6):427-30. 